KANSAS JOURNAL of MEDICINE

\section{Palbociclib-Induced Liver Failure}

Rami Atallah, M.D. ${ }^{1}$, Nathaniel A. Parker, D.O. ${ }^{1}$,

Karl Hamouche, M.D. ${ }^{2}$, Quoc V. Truong, M.D.,

Megan Dingwall, M.D. ${ }^{1}$

University of Kansas School of Medicine-Wichita, KS

'Department of Internal Medicine

${ }^{2}$ Department of Diagnostic Radiology

${ }^{3}$ Cancer Center of Kansas, Wichita, KS

Received May 28, 2019; Accepted for publication Dec. 23, 2019; Published online April 17, 2020

\section{INTRODUCTION}

In 2019, approximately 330,000 new cases of breast cancer are estimated to be diagnosed, with about $80 \%$ of those likely to be the invasive breast cancer type. ${ }^{1}$ On average, $75 \%$ of all newly diagnosed breast cancers are hormone receptor-positive $(\mathrm{HR}+){ }^{2}$ Although $\mathrm{HR}+$ breast cancer expresses both estrogen and progesterone receptors (ER; PR), ER signaling pathways ultimately determine cellular growth and survival. ${ }^{3}$ As a result, drug therapies were developed that selectively target vital steps in this ER signaling pathway and historically have been the foundation of HR-positive breast cancer treatment. As a group, these agents are termed endocrine therapy and include aromatase inhibitors (e.g., letrozole, anastrozole, and exemestane), selective ER modulators (tamoxifen), and selective ER down-regulators (fulvestrant).

Endocrine therapy alone has its limitations. Not only do certain breast cancer patients have inherent estrogen hormone resistance, but also HR+ tumor cells have the capability to use alternate, nonestrogen dependent pathways for further growth and survival., ${ }^{4,5}$ One such known alternate route is the cyclin D-cyclin-dependent kinase 4/6 (CDK4/6)-inhibitor of CDK4-retinoblastoma pathway. ${ }^{6}$ Recently, CDK4/6-inhibitors have been developed and investigated to be used alone or with traditional endocrine therapy for $\mathrm{HR}+$ breast cancer. $^{7-12}$

Palbociclib is an oral selective inhibitor of CDK4/6. This novel agent was awarded accelerated approval by the US Food and Drug Administration (FDA) in 2015 following the PALOMA-1 trial data showing that the combination of palbociclib with letrozole results in improved progression-free survival rates in metastatic, ER + , human epidermal growth factor receptor 2 (HER2)-negative postmenopausal breast cancer patients who had not had prior treatment for advanced disease. Subsequently, based on PALOMA-3 trial results, palbociclib also was approved for advanced breast cancer refractory to endocrine therapy when used in combination with fulvestrant. ${ }^{7}$ 14-18 This report presents a rare clinical case of fatal acute liver failure following administration of the novel CDK4/6-inihibor, palbociclib.

\section{CASE REPORT}

A 79-year-old female presented to the emergency department with acute encephalopathy and jaundice. Her past medical history was notable for stage IV invasive ductal carcinoma of the breast with bone metastasis, $\mathrm{ER}^{+}, \mathrm{PR}+$, HER2- by fluorescence in situ hybridization (FISH), who failed initial standard of care chemotherapy, and was started on palbociclib and fulvestrant (1st cycle: oral palbociclib 125 mg oral daily for days 1-21 of a 28-day cycle plus 7-day rest; 2 nd cycle: oral palbociclib $100 \mathrm{mg}$ daily for days 1-21 of a 28-day cycle plus 7-day rest; intramuscular fulvestrant $500 \mathrm{mg}$ on days 1, 15, and 29). In addition, her chronic medical conditions included type 2 diabetes mellitus, hyperlipidemia, and gastric esophageal reflux disease which were being managed with metformin $500 \mathrm{mg}$ daily, atorvastatin $10 \mathrm{mg}$ daily, and omeprazole $20 \mathrm{mg}$ daily, respectively.

The patient had completed her second cycle approximately one week prior to presentation. On admission, liver injury was evident based on lab results showing a total bilirubin of $4.6 \mathrm{mg} / \mathrm{dl}(1.0 \mathrm{mg} / \mathrm{dl}$ one month prior), aspartate transaminase of $200 \mathrm{u} / \mathrm{l}$ (AST, $50 \mathrm{u} / \mathrm{L}$ one month prior), alanine transaminase of $50 \mathrm{u} / \mathrm{l}$ (ALT 20 one month prior), alkaline phosphatase of $150 \mathrm{u} / \mathrm{l}(90 \mathrm{u} / \mathrm{L}$ one month prior), INR of 3.8, and ammonia of $70 \mathrm{u} / \mathrm{L}$. Serum analysis was negative for elevated acetaminophen. A thorough autoimmune serology and viral hepatitis workup for other possible causes of liver injury was nonrevealing. Thrombocytopenia and macrocytic anemia with equivocal support for hemolysis was present based on platelets of 35 x109/L, hemoglobin of $8.5 \mathrm{~g} / \mathrm{dL}$, mean corpuscular volume of $124 \mathrm{fL}$, lactic acid dehydrogenase of 940U/L (450 one month prior), fibrinogen of 170 $\mathrm{mg} / \mathrm{dL}$; D-dimer of 11,000 ng/mL, undetectable haptoglobin levels, and presence of schistocytes on peripheral smear analysis. Infectious workup revealed WBC of 7.5 x109/L, 22\% bands, 1 of 2 blood cultures positive for pansensitive Escherichia coli.

Roussel Uclaf Causality Assessment Method (RUCAM) ${ }^{19,20}$ and Drug-Induced Liver Injury Network (DILIN) ${ }^{21}$ severity scores for palbociclib were 8 (probable) and $5+$ (fatal), respectively. Biochemically, the liver injury was cholestatic (R factor of 0.2 ) with a marked increase in total bilirubin suggestive of bland cholestasis. Imaging studies showed a homogenously enlarged and lobulated liver surrounded by perihepatic free fluid. The right liver lobe tip extended inferiorly beyond the right kidney suggesting significant hepatomegaly (Figure 1). The gallbladder and biliary ducts were unremarkable. These findings were notable when compared to CT images of the abdomen performed two-years prior which was negative for hepatomegaly, cirrhosis, or a lobular appearance. Based on the patient's clinical history, presentation, and sonography, acute livery injury was more probable.

Despite standard of care therapies and steroids she developed refractory hypotension, her labs and encephalopathy worsened, and her overall condition deteriorated. The patient's liver enzymes and bilirubin continued to trend up: AST of $1268 \mathrm{u} / \mathrm{l}$, ALT of $167 \mathrm{u} / \mathrm{l}$, and total bilirubin of $9.8 \mathrm{u} / \mathrm{l}$. The patient subsequently received supportive treatment and transitioned to comfort care.

\section{DISCUSSION}

$\mathrm{HR}+$ breast cancer treatment guidelines support the combination of CDK-inhibitor plus endocrine therapy based on randomized control trial data showing that when used together these therapies are effective, delay tumor progression, and improve the rates of progression-free survival and overall survival. ${ }^{7-12}$ In the large clinical trials, adverse events 
were common and led to dose reductions and discontinuation of medications. However, there have been few case reports of clinically severe liver injury attributable to palbociclib.

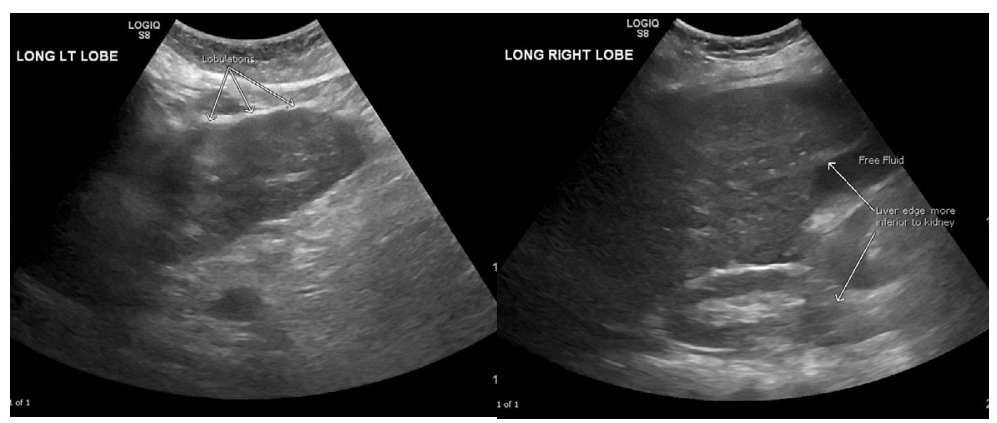

Figure 1. Ultrasound, liver. Left image: Homogenously enlarged liver with a grossly nodular and lobular appearance (arrows). Right image: Significant hepatomegaly represented by liver tip extending inferiorly beyond the level of the kidney, ascites (arrows).

Targeted therapies have revolutionized the landscape of oncologic treatments. Several studies have evaluated the safety and efficacy of palbociclib in combination with endocrine therapy. Elevated liver function tests were a rare but reported adverse event (AE) in 7.2\% of the palbociclib-treated patients in the PALOMA-1 study. ${ }^{22}$ In the PALOMA-2 study, ALT and AST elevations were reported as AEs (all grades) in $9.9 \%$ and $9.7 \%$ of palbociclib-treated patients, respectively. ${ }^{14}$ In the PALOMA-3 study, there was one fatal serious AE of hepatic failure with grade 5 disease progression in the palbociclib group; however, the patient's medical history included progressive liver metastasis and disease progression. ${ }^{8}$ Across all PALOMA studies, a pooled safety analysis demonstrated that grade $3 / 4$ AST and ALT elevations occurred in $3.3 \%$ and $2.3 \%$ of palbociclib-treated patients, respectively, again highlighting a reported but rare occurrence. ${ }^{23}$

Among all three PALOMA trials, neutropenia was the most frequently reported adverse reaction., ${ }^{7,14,16}$ Combination therapy of palbociclib with either letrozole or fulvestrant uncommonly resulted in grade 3 or 4 serious adverse events. The most frequently reported serious adverse reactions in patients who received palbociclib plus fulvestrant were infections, neutropenia, and pulmonary embolism. ${ }^{16}$ Among the 1,348 patients enrolled in the three PALOMA trials, none experienced fulminant hepatotoxicity that lead to stopping drug therapy. In the intention-to-treat populations, six cases of elevated AST $(n=1,1 \%)$, $\operatorname{ALT}(\mathrm{n}=3,1 \%), \gamma$-glutamyl transferase $(\mathrm{GGT}, \mathrm{n}=2,2 \%)$ were observed when palbociclib was administered in combination with letrozole. ${ }^{7.14}$ Ascites of any grade $(n=3,1.7 \%)$ was noted for patients receiving dual therapy of palbociclib plus fulvestrant. ${ }^{16}$ Thus, overall few treatmentrelated acute liver injuries were observed. However, final trial analysis of palbociclib plus fulvestrant noted one liver-related death that was not attributed to palbociclib. ${ }^{7}$ Moreover, the diagnosis of drug-induced liver injury remains challenging.

DILIN causality assessment process, despite its shortcomings, remains an important method in the field and a reasonable diagnostic standard. ${ }^{21}$ The mechanism of palbociclib-induced liver enzyme elevation is not well understood. Palbociclib is metabolized predominately by the hepatic CYP3A4 pathway. Thus, liver injury could be caused by the production of a toxic or immunogenic intermediate. Equally plausible, is coadministration with a strong CYP3A inhibitor, which increases the plasma exposure of palbociclib.
KANSAS JOURNAL of MEDICINE PALBOCICLIB-INDUCED LIVER FAILURE continued.

Open source software and databases show there would be a low likelihood of clinically significant drug-drug interactions between any of the patient's home medications and palbociclib. However, atorvastatin, metformin, and omeprazole are all CYP3A4 substrates and possible inhibitors. Thus, palbociclib metabolism theoretically could be inhibited causing increased serum concentrations of the substance. Currently, there is no official monitoring guidelines in terms of liver function testing when a patient is administered palbociclib therapy.

Likely based on PALOMA trial data, the palbociclib's FDA-approved product monograph only recommends monitoring for neutropenia by complete blood counts prior to starting palbociclib therapy, at the beginning of each cycle, and on day 14 of the first two cycles, as well as clinically indicated. ${ }^{24}$ No dose adjustment is required for patients with mild or moderate hepatic impairment. However, the recommended dose of palbociclib for patients with severe hepatic impairment (ChildPugh class C) is $75 \mathrm{mg}$ once daily. The patient detailed in this case had no history of hepatic impairment prior to starting treatment. Also, her liver function testing was unremarkable during assessments between cycles one and two of drug therapy.

In conclusion, the patient described in this case report illustrated an uncommon but serious complication from palbociclib in the treatment of metastatic breast cancer. Palbociclib should be considered as a possible cause of drug-induced liver injury when targeted CDK 4/6based regimens are used. ${ }^{23}$ Serum aminotransferase elevations above 3-to-5 times the upper limit of normal or any elevations accompanied by jaundice should alarm physicians and lead to temporary or permanent cessation of palbociclib therapy.

\section{REFERENCES}

${ }^{1}$ Breastcancer.org. U.S. Breast Cancer Statistics. February 13, 2019. https:/ www.breastcancer.org/symptoms/understand_bc/statistics. Accessed April 10, 2019.

${ }^{2}$ Hosford SR, Miller TW. Clinical potential of novel therapeutic targets in breast cancer: CDK4/6, Src, JAK/STAT, PARP, HDAC, and PI3K/AKT/ mTOR pathways. Pharmgenomics Pers Med 2014; 7:203-215. PMID: 25206307.

${ }^{3}$ Sammons SL, Topping DL, Blackwell KL. HR+, HER2- advanced breast cancer and CDK4/6 inhibitors: Mode of action, clinical activity, and safety profiles. Curr Cancer Drug Targets 2017; 17(7):637-649. PMID: 28359238.

4 García-Becerra R, Santos N, Díaz L, Camacho J. Mechanisms of resistance to endocrine therapy in breast cancer: Focus on signaling pathways, miRNAs and genetically based resistance. Int J Mol Sci 2012; 14(1):108-145. PMID: 23344024.

${ }^{5}$ Osborne C, Schiff R. Mechanisms of endocrine resistance in breast cancer. Annu Rev Med 2011; 62:233-247. PMID: 20887199.

${ }^{6}$ Lukas J, Bartkova J, Bartek J. Convergence of mitogenic signalling cascades from diverse classes of receptors at the cyclin D-cyclin-dependent kinase-pRb-controlled Gl checkpoint. Mol Cell Biol 1996; 16(12):69176925. PMID: 8943347.

Finn R, Martin M, Rugo HS, et al. Palbociclib and letrozole in advanced breast cancer. N Engl J Med 2016; 375(20):1925-1936. PMID: 27959613.

${ }^{8}$ Cristofanilli M, Turner NC, Bondarenko I, et al. Fulvestrant plus palbociclib versus fulvestrant plus placebo for treatment of hormone-receptor-positive, HER2-negative metastatic breast cancer that progressed on previous endocrine therapy (PALOMA-3): Final analysis of the multicentre, double-blind, phase 3 randomised controlled trial. Lancet Oncol 2016; 17(4):425-439. PMID; 26947331. 


\section{KANSAS JOURNAL of MEDICINE}

PALBOCICLIB-INDUCED LIVER FAILURE

continued.

9 Hortobagyi GN, Stemmer SM, Burris HA, et al. Ribociclib as first-line therapy for HR-positive, advanced breast cancer. N Engl J Med 2016; 375(18):1738-1748. PMID: 27717303.

${ }_{10}$ Torres R, Calsina B, Hermoso A, et al. Characterization of the mechanism of action for abemaciclib with antiestrogen combined therapy in human breast cancer cell lines. [Abstract.] Cancer Res 2016; 76(14 Suppl):Abstract nr 2836.

${ }^{11}$ Dickler MN, Tolaney SM, Rugo HS, et al. J. MONARCH 1: Results from a phase II study of abemaciclib, a CDK4 and CDK6 inhibitor, as monotherapy, in patients with $\mathrm{HR}+/ \mathrm{HER} 2$ - breast cancer, after chemotherapy for advanced disease. J Clin Oncol 2016; 34(15 Suppl): 510.

${ }^{12}$ National Comprehensive Cancer Network. NCCN Clinical Practice Guidelines in Oncology. Breast Cancer, version 2.2016. http://www.ncen. org. Accessed April 10, 2019.

${ }^{13}$ Vuppalanchi R, Saxena R, Storniolo AMV, Chalasani N. Pseudocirrhosis and liver failure in patients with metastatic breast cancer after treatment with palbociclib. Hepatology 2017; 65(5):1762-1764. PMID: 27397671.

${ }^{14}$ Finn RS, Crown JP, Lang I, et al. The cyclin-dependent kinase 4/6 inhibitor palbociclib in combination with letrozole versus letrozole alone as first-line treatment of oestrogen receptor-positive, HER2-negative, advanced breast cancer (PALOMA-1/TRIO-18): A randomised phase 2 study. Lancet Oncol 2015; 16(1):25-35. PMID: 25524798.

${ }_{15}$ US Food and Drug Administration. Palbociclib (Ibrance). https://www. fda.gov/drugs/resources-information-approved-drugs/palbociclib-ibrance. March 31, 2017. Accessed April 6, 2019.

${ }_{16}$ Verma S, Bartlett CH, Schnell P, et al. Palbociclib in combination with fulvestrant in women with hormone receptor-positive/HER2-negative advanced metastatic breast cancer: Detailed safety analysis from a multicenter, randomized, placebo-controlled, phase III study (PALOMA-3). Oncologist 2016; 21(10):1165-1175. PMID: 27368881.

${ }_{17}$ Finn RS, Martin M, Rugo HS, et al. Revised Supplementary Appendix: Palbociclib and Letrozole in Advanced Breast Cancer. Table S3 Dose Reductions and Interruptions. https://www.nejm.org/doi/suppl/10.1056/ NEJMoal607303/suppl_file/nejmoal607303_appendix.pdf. Accessed June 5, 2019.

18 Verma S, Bartlett CH, Schnell P, et al. Supplemental Figures: Palbociclib in combination with fulvestrant in women with hormone receptor-positive/ HER2-negative advanced metastatic breast cancer: Detailed safety analysis from a multicenter, randomized, placebo-controlled, phase 3 study (PALOMA-3). Table Sl. Summary of treatment-emergent serious adverse events (> case, all causalities): as-treated population. http://theoncologist. alphamedpress.org/content/suppl/2016/07/01/theoncologist.2016-0097. DCl/Supplemental_Tables.pdf. Accessed June 5, 2019.

19 Danan G, Benichou C. Causality assessment of adverse reactions to drugs-I. A novel method based on the conclusions of International Consensus Meeting: Application to drug-induced liver injuries. J Clin Epidemiol 1993; 46(11):1323-1130. PMID: 8229110.

${ }^{20}$ Benichou C, Danan G, Flahault A. Causality assessment of adverse reactions to drugs-II. An original model for validation of drug causality assessment methods: Case reports with positive rechallenge. J Clin Epidemiol 1993; 46(11):1331-1336. PMID: 8229111

${ }^{21}$ Hayashi PH. Drug-Induced Liver Injury Network causality assessment: Criteria and experience in the United States. Int J Mol Sci 2016; 17(2):201. PMID: 26861284.

22 Turner NC, Ro J, André F, et al. Palbociclib in hormone-receptor-positive advanced breast cancer. N Engl J Med 2015; 373(3):209-219. PMID: 26030518

${ }^{23}$ Roberts BA, Ibrahim M, Stone E. Elevated liver function test in a patient on palbociclib and fulvestrant. J Community Support Oncol 2018; 16(6):e277-e279.

${ }^{24}$ Palbociclib [product monograph]. Kirkland, Quebec: Pfizer Canada ULC, 2019.

Keywords: palbociclib, acute hepatic failure, hepatic encephalopathy 